Original article

\title{
Effects of amphetamine, diazepam and caffeine on polysomnography (EEG, EMG, EOG)-derived variables measured using telemetry in Cynomolgus monkeys ${ }^{\text {i⿱ }}$
}

\author{
Simon Authier ${ }^{\mathrm{a}, \mathrm{b}, *, 1}$, Leanne Bassett ${ }^{\mathrm{a}, \mathrm{b}, 1}{ }$, Mylene Pouliot ${ }^{\mathrm{a}}$, Adeline Rachalski ${ }^{\mathrm{c}}$, Eric Troncy ${ }^{\mathrm{b}}$, \\ Dominique Paquette ${ }^{\mathrm{a}}$, Valérie Mongrain ${ }^{\mathrm{c}, \mathrm{d}}$ \\ a CIToxLAB North America, 445 Armand Frappier, Laval, QC, Canada, H7V $4 B 3$ \\ ${ }^{\mathrm{b}}$ Faculty of Veterinary Medicine, University of Montreal, P.O. box 5000, St-Hyacinthe, QC, Canada, J2S 7C6 \\ c Center for Advanced Research in Sleep Medicine and Research Center, Hôpital du Sacré-Coeur de Montréal, Montreal, QC, Canada, H4J 1C5 \\ d Department of Neuroscience, Université de Montréal, Montreal, QC, Canada, H3C 3J7
}

\section{A R T I C L E I N F O}

\section{Article history:}

Received 12 March 2014

Accepted 20 May 2014

Available online 27 May 2014

\section{Keywords:}

Animal model

EEG spectral analysis

Macaca fascicularis

Pharmacology

Sleep stages

Telemetry

\begin{abstract}
A B S T R A C T
Introduction: Medication-induced sleep disturbances are a major concern in drug development as a multitude of prescription drugs alter sleep patterns, often negatively. Polysomnography is used in clinical diagnostics but is also applicable to animal models. Rodent sleep architecture (nocturnal) differs from larger diurnal mammals, including humans, increasing the translational potential of non-rodent species to the clinic. This study aimed to characterize the response to pharmacological agents known to affect sleep structure and EEG activity in a non-human primate (Macaca fascicularis) using telemetry-based polysomnography. Methods: Animals were instrumented with telemetry transmitters for continuous electroencephalogram (EEG), electro-oculogram (EOG) and electromyogram (EMG) monitoring combined with video. EEG, EMG and EOG were monitored for 12 to $24 \mathrm{~h}$ to establish baseline values, followed by administration of pharmacological agents (saline, d-amphetamine, diazepam or caffeine). Results: Amphetamine $(0.3$ and $1 \mathrm{mg} / \mathrm{kg}$, by oral administration (PO)) significantly reduced total sleep time, including the duration of both non-rapid eye movement [NREM] sleep and REM sleep. It also decreased EEG activity in low frequencies (i.e., 4-6 Hz) during wakefulness. Diazepam ( $2 \mathrm{mg} / \mathrm{kg}$, PO) did not significantly alter sleep duration, but importantly reduced EEG activity in low frequencies (approximately $2-12 \mathrm{~Hz}$ ) during wakefulness, NREM and REM sleep. Finally, caffeine (10 and $30 \mathrm{mg} / \mathrm{kg}$, PO) decreased both NREM and REM sleep duration. In addition, spectral analysis revealed important decreases in low frequency activity (i.e., 1-8 Hz) during wakefulness with a parallel increase in high frequency activity (i.e., 20-50 Hz) during NREM sleep. Discussion: As these observations are similar to previously reported pharmacological effects in humans, results support that EEG, EOG and EMG monitoring by telemetry in Cynomolgus monkeys represents a useful non-clinical model to investigate and quantify drug-induced sleep disturbances.
\end{abstract}

(c) 2014 Elsevier Inc. All rights reserved.

\section{Introduction}

A number of medications, including several widely used drugs such as nicotine replacement therapy (Mills, Wu, Lockhart, Wilson, \& Ebbert, 2010), oral contraceptives (Baker, Mitchell, \& Driver, 2001), aspirin and ibuprofen (Murphy, Badia, Myers, Boecker, \& Wright, 1994) have been shown to impact on objective and subjective sleep quality in humans.

\footnotetext{
F Funding: The research was conducted thanks to a NSERC grant (386623-2010, VM), a FRQS salary award (22210, VM), start-up funds from the CR-HSCM (VM), and CiToxLAB North America.

* Corresponding author at: CIToxLAB North America, 445 Armand Frappier, Laval, Quebec, Canada, H7V 4B3.

E-mail address: authiers@ca.citoxlab.com (S. Authier).

1 Simon Authier and Leanne Bassett have contributed equally to this manuscript.
}

Other drugs including beta-blockers, benzodiazepines and opioids were also shown to affect sleep architecture. Indeed, beta-blockers, such as propranolol, metoprolol and pindolol increase wake after sleep onset and reduce rapid eye movement (REM) sleep (Betts \& Alford, 1985). Benzodiazepines decrease sleep latency, usually defined as the time elapsed from lights off to the first minute of sleep, and increase total sleep time, but also affect the proportion of the different sleep stages, by increasing time in non-rapid eye movement (NREM) sleep stage 2 and decreasing NREM sleep stage 3 and REM sleep, therefore reducing deep sleep (Buscemi et al., 2005; Carlstedt, 2009). Opioids disturb sleep and decrease duration of NREM sleep stage 2 and REM sleep (Ramakrishnan \& Scheid, 2007). The reduction in sleep efficiency, which takes into account the time spent awake during the sleep period, by drugs such as caffeine and d-amphetamine has been widely reported 
(Bonnet \& Arand, 1992; Boutrel \& Koob, 2004). In addition, most pharmacological substances used as sleep medication or to treat psychiatric disorders lead to modifications of the spectral profile of sleep and wake states (Hasan et al., 2009; Paterson, Wilson, Nutt, Hutson, \& Ivarsson, 2009; Schmid et al., 2006). The European Medicines Agency (2011) confirms that multichannel polysomnography is a valuable tool that enables objective assessment of sleep architecture, including sleep latency, number of awakening and total sleep time in the context of drug development for sleep disorders. EEG spectral analysis thus needs to be used to refine the characterization of drug-induced alterations in sleep.

During pharmacological development, identification of potential effects on sleep duration and architecture as well as on EEG activity during sleep can impact on safety and consequently, on prescription rate. Reduced sleep duration (e.g., sleep deprivation) has been shown to be detrimental to human health and can contribute to diabetes (Gottlieb et al., 2005), various hormonal deficiencies (Vgontzas et al., 1999) as well as neurological disorders (Palma, Urrestarazu, \& Iriarte, 2013), emphasizing the importance of managing the risk associated with changes in sleep architecture in drug development. Slow wave characteristics of deep sleep (i.e., NREM sleep stage 3) have also been hypothesized to directly mediate recovery during sleep (Tononi \& Cirelli, 2006). Pre-clinical models to investigate potential adverse effects of drugs on sleep as well as assessment of the efficacy of drugs that may improve quality and duration of sleep are thus necessary (Schmid et al., 2006).

In humans, sleep and wakefulness episodes are mostly consolidated in adults (Carskadon \& Dement, 2011), and sleep usually occupies a third of the nycthemeron. A night of sleep usually begins with a short period of NREM sleep stage 1 (N1), which is followed by NREM sleep stage 2 (N2) characterized by the presence of sleep spindles and $\mathrm{K}$-complexes. These stages are usually followed by NREM sleep stage 3 (N3) which is mostly composed of slow waves that are indexed by EEG slow wave activity (SWA, 1-5 Hz). Episodes of REM sleep, characterized by muscle atonia and intermittent REM observable on the electro-oculogram (EOG), become increasingly longer as the night progresses. Stages N1, N2, N3 and REM generally represent 2-5\%, 45-55\%, $10-20 \%$ and $20-25 \%$ of total sleep time in humans, respectively. In rodents, however, sleep is polyphasic with very frequent transitions between wakefulness and sleep in addition to occurring mostly during the light period (Franken, Malafosse, \& Tafti, 1998; Van Twyver, 1969). Moreover, characterization of the sleep/wake cycle in mice includes wakefulness, NREM sleep and REM sleep, with NREM sleep representing close to $90 \%$ of total sleep time (C57BL/6J strain; Franken et al., 1998). As such, the mouse model lacks the different NREM sleep stages identified in humans, which may reduce sensitivity in some cases. Rodent models thus present some limitations when investigating potential drug effects on sleep duration or architecture. Conversely, Cynomolgus monkeys are diurnal and show similar proportion of sleep stages in comparison to humans (Porsolt, 2013; Rachalski et al., in press).

The fact that Cynomolgus monkeys are diurnal mammals like humans emphasizes the appropriateness of this non-human primate as a translational model of human sleep because it suggests a similar regulation of sleep by the circadian timing system. The circadian system, controlled by the suprachiasmatic nuclei (SCN) of the hypothalamus, regulates many physiological functions including the sleep-wake cycle, hormonal secretion and metabolism (Kriegsfeld, LeSauter, Hamada, Pitts, \& Silver, 2002; Kriegsfled \& Silver, 2006). In addition, circadian control of hormone secretion can influence sleep, which is particularly true for melatonin that also directly influences SCN activity (Kriegsfled \& Silver, 2006). Similar to humans, diurnal nonhuman primates have elevated melatonin production during the main rest period (i.e., the dark phase), and melatonin administration favors sleep (Zhdanova et al., 2002) thus supporting their translational potential.
Scarce data are available on sleep architecture in macaque monkeys (Barraud et al., 2009; Hsieh, Robinson, \& Fuller, 2008). Prolonged physical restraint during EEG monitoring (Pigarev, Almirall, \& Pigareva, 2008), along with limited length of recording sessions characterizes many of the data sets previously published (Benca, Obermeyer, Shelton, Droster, \& Kalin, 2000). The use of telemetry EEG monitoring enables animals to freely move allowing for the assessment of the animal's natural sleep architecture (Hsieh et al., 2008; Rachalski et al., in press). Accordingly, the current study aimed to validate the use of telemetry to investigate the effects of different pharmacological treatments on polysomnography (EEG, EOG and EMG)-derived variables, including EEG spectral activity, in unrestrained Cynomolgus monkeys.

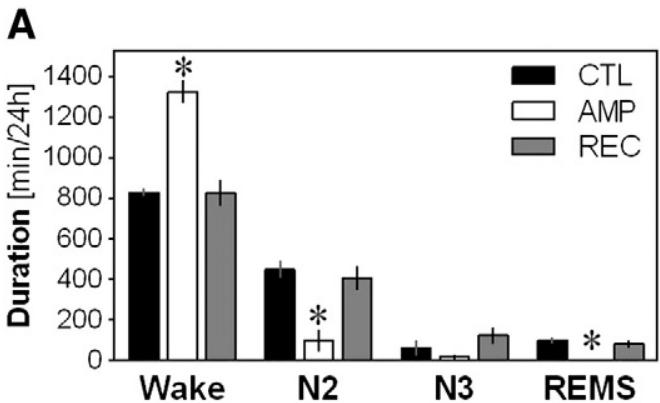

B

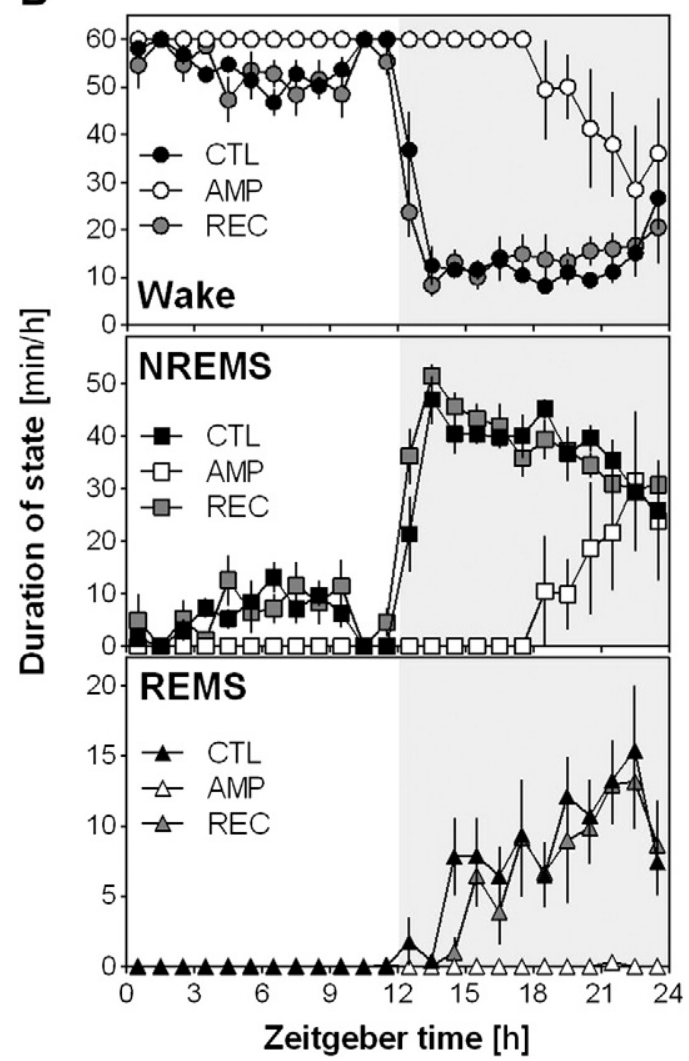

Fig. 1. Duration and distribution of vigilance states in Cynomolgus monkeys under control treatment, d-amphetamine ( $1+0.56 \mathrm{mg} / \mathrm{kg}, 9 \mathrm{~h}$ apart), and recovery. A) Total duration of wakefulness (wake), N2, N3 and REM sleep (REMS) for a 24-hour period averaged for 4 monkeys. D-amphetamine significantly changed wakefulness, N2 and REMS $\left(F_{2,6}>23.3\right.$, $p<0.01$ ). Stars indicate Tukey HSD $<0.01$ compared to both control and recovery. B) Hourly distribution of wakefulness, NREM sleep (NREMS: N1 to N3) and REMS in control condition, under d-amphetamine $(1+0.56 \mathrm{mg} / \mathrm{kg}, 9 \mathrm{~h}$ apart $)$ and during recovery in 4 animals. Zeitgeber time refers to the time relative to the light/dark schedule with 0 corresponding to lights ON and 12 to lights OFF. Grey backgrounds indicate the dark period. 


\section{Materials and methods}

\subsection{Statement on use and care of animals}

During the study, care and use of animals were conducted in accordance with principles outlined in the current Guide to the Care and Use of Experimental Animals published by the Canadian Council on Animal Care and the Guide for the Care and Use of Laboratory Animals published by the US National Institutes of Health (NIH Publication No. 85-23, revised 2010). CiToxLAB North America's facility is AAALAC accredited. All procedures were conducted as per Standard Operating Procedures (SOPs).

\subsection{Animal housing and preparation}

Nine (9) male Cynomolgus monkeys (Macaca fascicularis) were surgically prepared with telemetry transmitters (TL11M2-D70-EEETM, DSI, St-Paul, MN, USA) as described previously (Authier et al., 2009). Monkeys were 2.5 to 3 years old and weighed between 2.6 and $3.7 \mathrm{~kg}$. The animal room environment was controlled (temperature $21 \pm 3{ }^{\circ} \mathrm{C}$, humidity 30-70\%, $12 \mathrm{~h}$ light, $12 \mathrm{~h}$ dark, 10-15 air changes per hour) with temperature and relative humidity being monitored continuously. A standard certified commercial primate chow (Certified Hi-Fiber Primate Diet $7195 C^{\mathrm{TM}}$, Harlan Teklad, Madison, WI, USA) was available to each monkey twice daily. A prophylactic antibiotic (Cefazolin, Novopharm, Toronto, ON, Canada, $30 \mathrm{mg} / \mathrm{kg}$ ) was administered by intravenous (IV) injection at least 15-20 min prior to surgery and every $2 \mathrm{~h}$ postinjection for a maximum of 3 doses. Preemptive analgesia was attained via a transdermal Fentanyl patch (Sandoz, Boucherville, QC, Canada, $12.5 \mu \mathrm{g} / \mathrm{h}$ ) placed on the animal the day prior to surgery and removed 2 days post-surgery. A non steroidal anti-inflammatory (Meloxicam, Boehringer Ingelheim, Burlington, ON, Canada, $0.1 \mathrm{mg} / \mathrm{kg}$, SID, SC) was administered for 3 days after surgery. A local anesthetic (Marcaine, Hospira, Montreal, QC, Canada, $0.4 \mathrm{~mL}$; Lidocaine, Vetoquinol, Lavaltrie, QC, Canada; $20 \mathrm{mg} / \mathrm{mL}, 0.4 \mathrm{~mL}$ ) was injected subcutaneously in 6-10 sites distributed over the neck, the cranial, abdominal and orbital surgical sites during the surgery. Animals were placed on a heating pad and inhaled a mixture of oxygen $\left(\mathrm{O}_{2}\right)$ and isoflurane (AErrane ${ }^{\mathrm{TM}}$, Baxter Corporation, Mississauga, ON, CAN) with the $\mathrm{O}_{2}$ flow meter and the vaporizer set at $1.0 \mathrm{~L} / \mathrm{min}$ and $2.0 \%$, respectively. Respiratory rate was maintained between 12 and 15 breaths/min with an inspiratory airway pressure between 18 and $20 \mathrm{~cm} \mathrm{H}_{2} \mathrm{O}$ using a mechanical ventilator (Hallowell EMC, Pittsfield, MA, USA). During anesthesia, body temperature and end-tidal $\mathrm{CO}_{2}$ were monitored continuously. A longitudinal incision was performed lateral but close to the linea alba, and the internal abdominal oblique muscle was separated from the aponeurosis of the transverse abdominus. The telemetry transmitter was placed between the internal abdominal oblique muscle and the aponeurosis of the transversus abdominis muscle. The rectus abdominis was sutured with a simple continuous suture and electroencephalographic (EEG) electrodes were tunnelled subcutaneously to a small skin incision in the neck. The abdominal skin incision was closed with interrupted buried sutures and the animal was placed in sternal recumbency to expose the cranium for the remainder of the surgery. EEG leads were placed on the cranium to monitor one standard bipolar derivation $(\mathrm{Cz}-\mathrm{Oz}) . \mathrm{A}$ linear groove was made in the cranial cortical bone to secure the electrodes with surgical glue (Vetbond ${ }^{\mathrm{TM}}, 3 \mathrm{M}$, St-Paul, MN, USA) and acrylic. The electromyographic (EMG) electrodes were positioned parallel to the longitudinal axis of the neck muscles with a distance of $10 \mathrm{~mm}$ between leads. Electro-oculographic (EOG) leads were placed subcutaneously below the outer canthus and above the inner canthus of the right eye and sutured in the periosteum. At completion of surgery, one drop of Ciloxan 0.3\% (Alcon Canada Inc., Mississauga, ON, Canada) was applied to both eyes. A period of 4 weeks was allowed between the
A

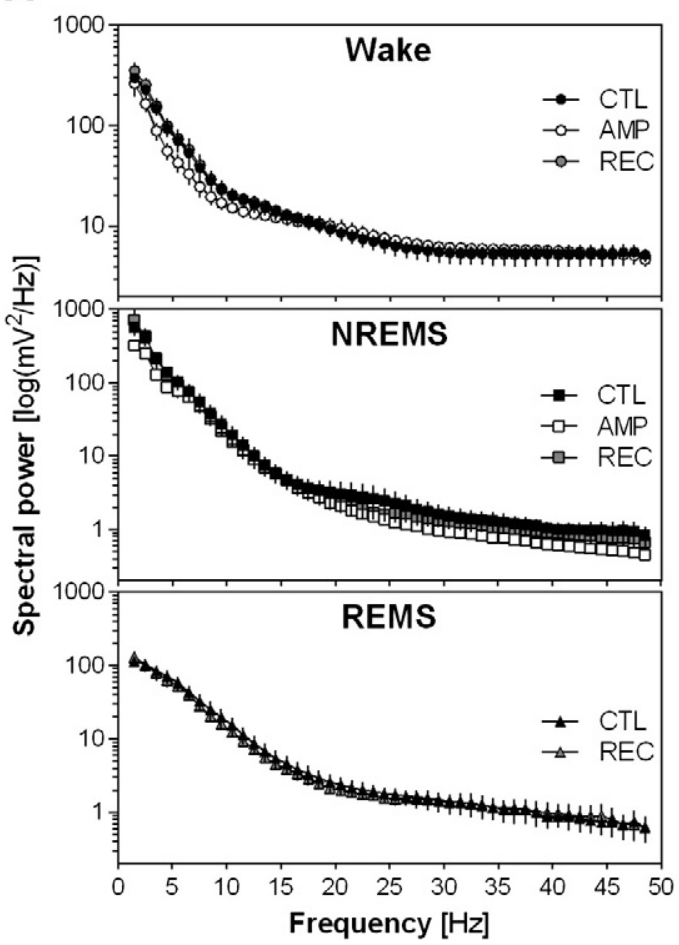

B

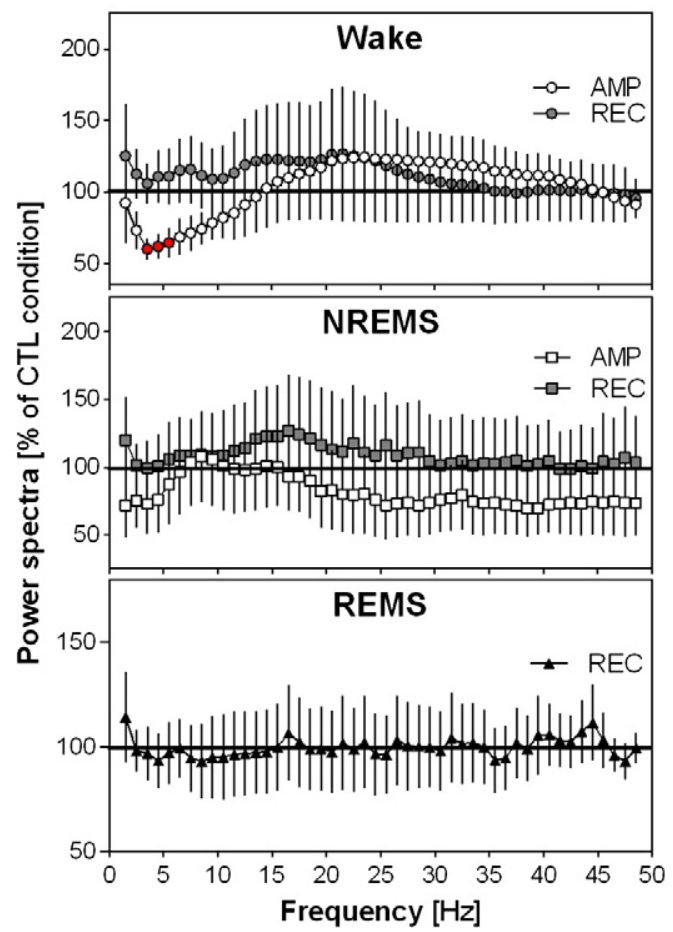

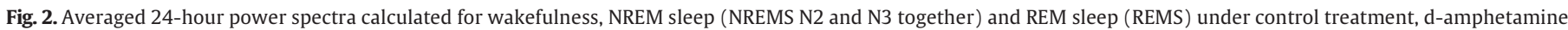

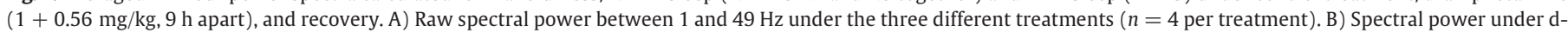

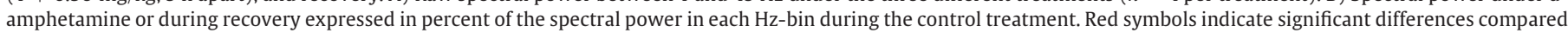
to the control condition ( $t$-test: $p<0.05)$. 
surgery and the start of experimental procedures which included administration of saline, amphetamine, diazepam and caffeine as described in subsequent sections.

\subsection{Polysomnography and pharmacological treatments}

\subsubsection{Electrophysiological and behavioural monitoring}

The EEG, EMG and EOG monitoring included a digital color camera with daylight and infrared night vision connected to a computerized system (IBM Intellistation Z pro, Xeon 3.8 Ghz, 3.5 TB hard drive). The video recording was used as a complement for interpretation of EEG traces when necessary. Acquisition of the telemetry signal was performed at a sampling rate of $500 \mathrm{~Hz}$ (Ponemah version 4.8, DSI,

A

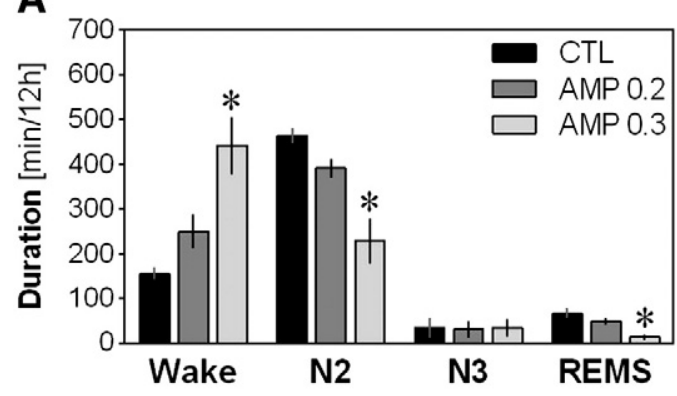

B

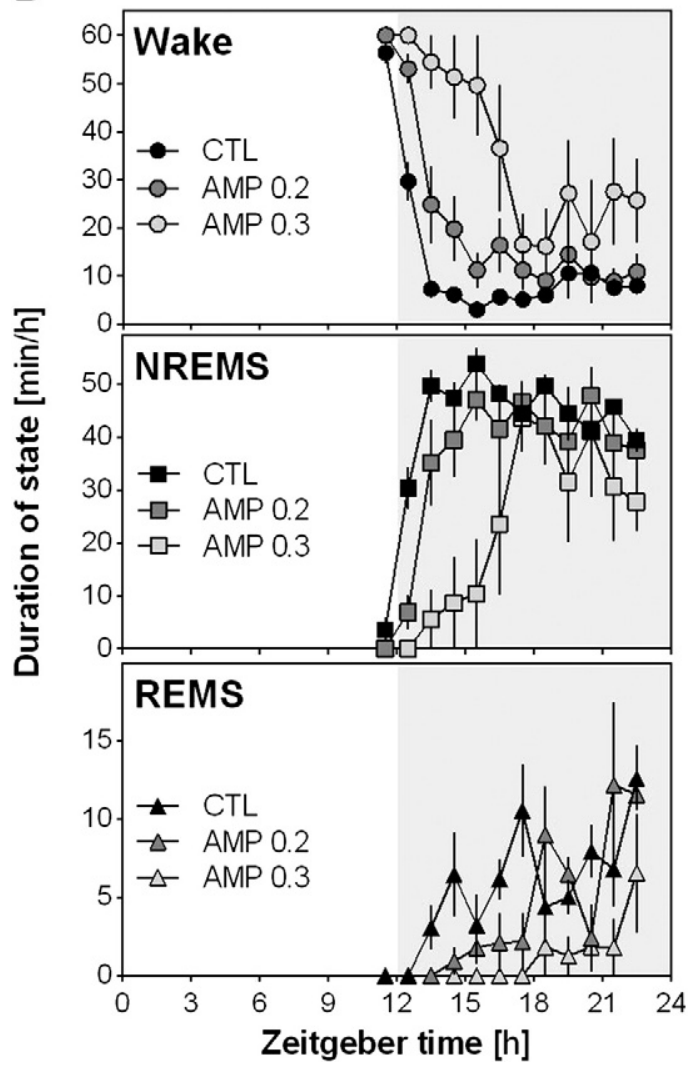

Fig. 3. Duration and distribution of vigilance states in Cynomolgus monkeys under control treatment or two doses of d-amphetamine $(0.2$ and $0.3 \mathrm{mg} / \mathrm{kg})$. A) Total duration of wakefulness, N2, N3 and REM sleep (REMS) for a 12-hour period averaged for 5 monkeys. D-amphetamine significantly changed wakefulness, N2 and REMS $\left(\mathrm{F}_{2,6}>7.2, p<0.05\right)$. Stars indicate Tukey HSD $<0.05$ compared to control. B) Hourly distribution of wakefulness, NREM sleep (NREMS: N1 to N3) and REMS in control condition, and under d-amphetamine $(0.2$ and $0.3 \mathrm{mg} / \mathrm{kg})$. Zeitgeber time refers to the time relative to the light/dark schedule with 0 corresponding to lights ON and 12 to lights OFF. Grey backgrounds indicate the dark period.
St-Paul, MN). EEG, EMG and EOG were monitored continuously for $24 \mathrm{~h}$ starting at light onset to establish baseline prior to dosing until at least $48 \mathrm{~h}$ following dosing for d-amphetamine $(1 \mathrm{mg} / \mathrm{kg})$ administration. For the portion of the study in which diazepam, caffeine or d-amphetamine $(0.2$ and $0.3 \mathrm{mg} / \mathrm{kg})$ was administered, EEG, EMG and EOG were monitored continuously for $1 \mathrm{~h}$ prior to dose administration until at least $12 \mathrm{~h}$ following dosing.

\subsection{2. d-Amphetamine}

d-Amphetamine (GlaxoSmithKline) was administered to four (4) male Cynomolgus monkeys PO twice. The first dose was $1 \mathrm{mg} / \mathrm{kg}$ $(0.2 \mathrm{mg} / \mathrm{mL})$ provided at approximately 08:00 (light onset), and the second was of $0.56 \mathrm{mg} / \mathrm{kg}$ at approximately 17:00 (9 h after light onset). Treatment was provided in a cross over design with the control treatment. In a given treatment session, half of the animals received
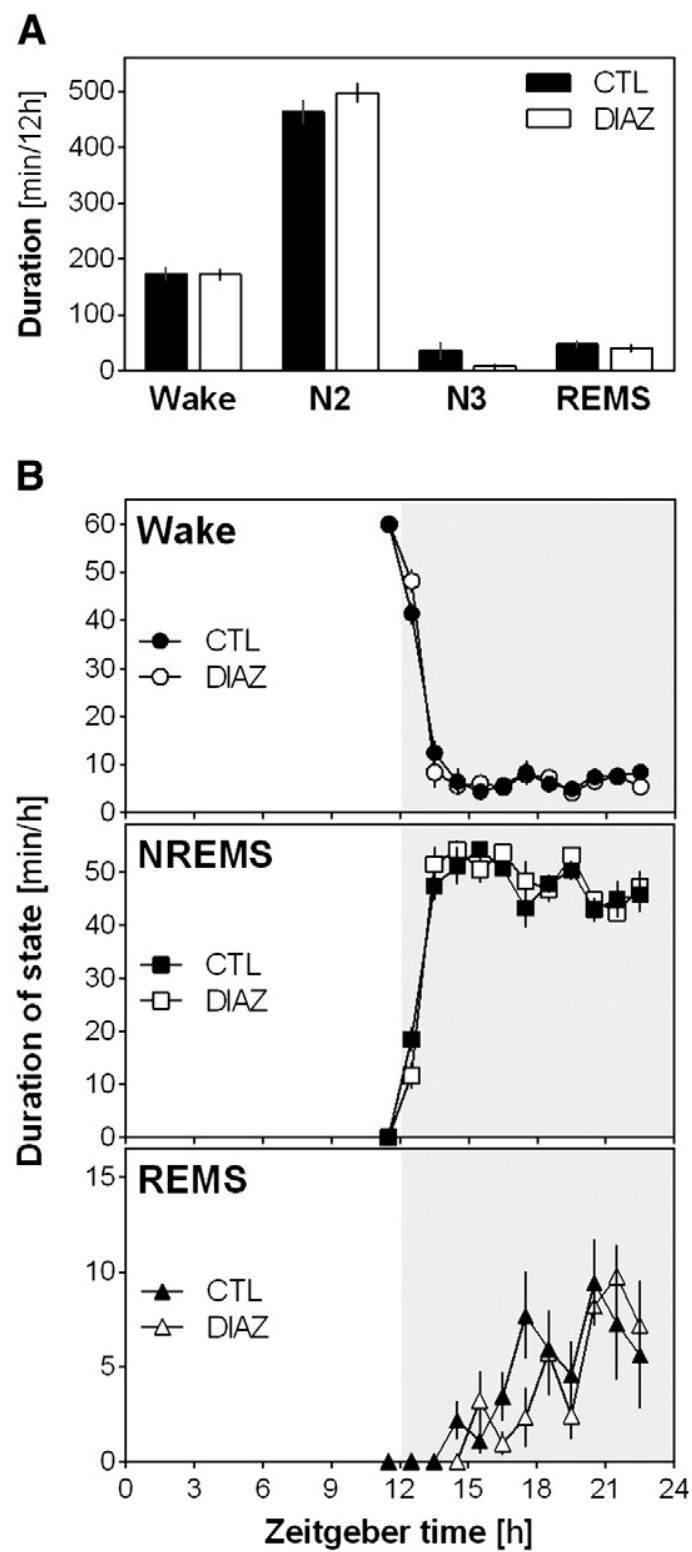

Fig. 4. Duration and distribution of vigilance states in Cynomolgus monkeys under control treatment or diazepam ( $2 \mathrm{mg} / \mathrm{kg}$ ). A) Total duration of wakefulness, N2, N3 and REM sleep (REMS) for a 12-hour period averaged for 6 monkeys. B) Hourly distribution of wakefulness, NREM sleep (NREMS: N1 to N3) and REMS in control condition and under diazepam administration $(n=6)$. Zeitgeber time refers to the time relative to the light/dark schedule with 0 corresponding to lights ON and 12 to lights OFF. Grey backgrounds indicate the dark period. 
d-amphetamine and half received the control. A period of at least 7 days was allowed between each treatment. A second cohort of five (5) male Cynomolgus monkeys received d-amphetamine at a dose level of 0.2 and $0.3 \mathrm{mg} / \mathrm{kg}$ PO approximately $10 \mathrm{~min}$ prior to the scheduled lights off, in a cross over design with the control treatment.

\subsubsection{Diazepam}

Control and diazepam ( $2 \mathrm{mg} / \mathrm{kg}, \mathrm{PO})$ were administered in a cross over design to six (6) male Cynomolgus monkeys. Treatments were administered up to 30 min prior to the scheduled lights off.

\subsubsection{Caffeine}

Caffeine (3, 10 and $30 \mathrm{mg} / \mathrm{kg}, \mathrm{PO})$ was administered approximately 10 min prior to the scheduled lights off to five (5) male Cynomolgus monkeys in a cross over design with control treatment.

\subsection{EEG analysis methods}

Trace analyses of EEG, EMG and EOG were carried out using NeuroScore software Version 1.1 (DSI, St-Paul, MN, USA) similar to gold standard techniques employed in humans (Campbell, (2009). Sleep stages were visually attributed per 10 -sec epoch by trained technicians using the criteria described in Rachalski et al. (in press). Briefly, wake was characterized by low amplitude and high frequency EEG; stage N1 by slower EEG activity (generally $4-8 \mathrm{~Hz}$ ) and higher amplitude than during wake; stage $\mathrm{N} 2$ by the presence of characteristic sleep spindles (regular 12-16 Hz EEG sequences lasting at least $0.5 \mathrm{~s}$ ) and $\mathrm{K}$ complexes (1-4 Hz isolated waves including a negative sharp wave followed by a positive component); stage N3 by the presence of high amplitude $(>75 \mu \mathrm{V})$ slow EEG waves $(1-4 \mathrm{~Hz})$; and REM sleep by the predominance of regular 4-8 Hz EEG activity accompanied with EMG atonia. Traces from a given animal were only analyzed by a single designated trained technician to minimize inter-scorer variations in the characterization of drug effects. The time spent in the different sleep stages (N1, N2, N3 and REM) was calculated over $24 \mathrm{~h}, 12 \mathrm{~h}$ or per hour. Spectral analysis was performed using fast Fourier transform (FFT) for all 10-sec epochs, and activity of artefact-free epochs from the different stages was averaged to quantify absolute EEG activity between 1 and $50 \mathrm{~Hz}$ per $1-\mathrm{Hz}$ bin. The acquisition system included a high pass filter at $1 \mathrm{~Hz}$. FFT enables quantification of the power at each frequency within the 10 -sec epoch by separating the EEG signal into a series of sine and cosine waves (Chen \& Black, 2005). EEG spectra were also expressed relative to each animal control treatment. Results are reported as mean \pm SEM.

\section{Results}

The duration of each sleep stage obtained from baseline data prior to the administration of any pharmacological agent revealed that total sleep time was $70.3 \pm 2.2 \%$ of the 12 -hour dark period. Sleep stages $\mathrm{N} 1, \mathrm{~N} 2, \mathrm{~N} 3$ and REM were typically observed in this sequence and represented $1.1 \pm 0.3 \%, 65.2 \pm 5.3 \%, 16.0 \pm 5.6 \%$ and $17.7 \pm 2.4 \%$ of total sleep time, respectively.

\section{1. d-Amphetamine}

The administration of d-amphetamine $(1+0.56 \mathrm{mg} / \mathrm{kg}, 9 \mathrm{~h}$ apart $)$ significantly enhanced wakefulness duration during a 24 hour recording (Fig. 1A). This was accompanied with a significant decrease in N2 and REM sleep. Elevated wakefulness and reduced sleep were observed for most of the 24 hour period with only the last two recording hours showing normalized wakefulness and NREM sleep amount, while REM sleep remained virtually absent (Fig. 1B). All these effects on sleep stage duration were completely recovered during the $24 \mathrm{~h}$ following d-amphetamine administration (Fig. 1A and 1B), and thus no apparent compensatory effect was observed during the second day following
A

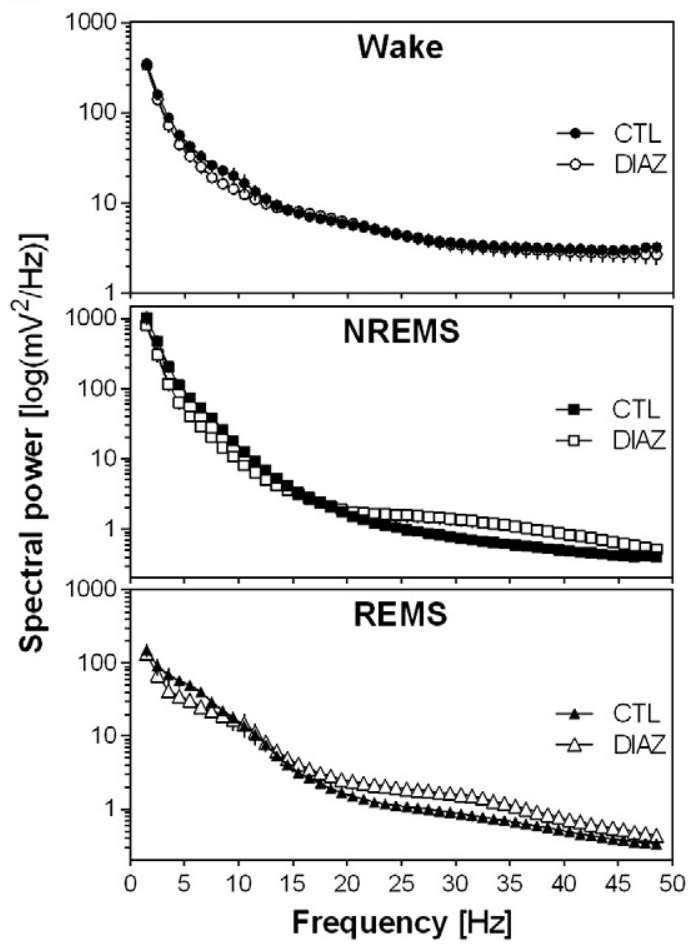

B

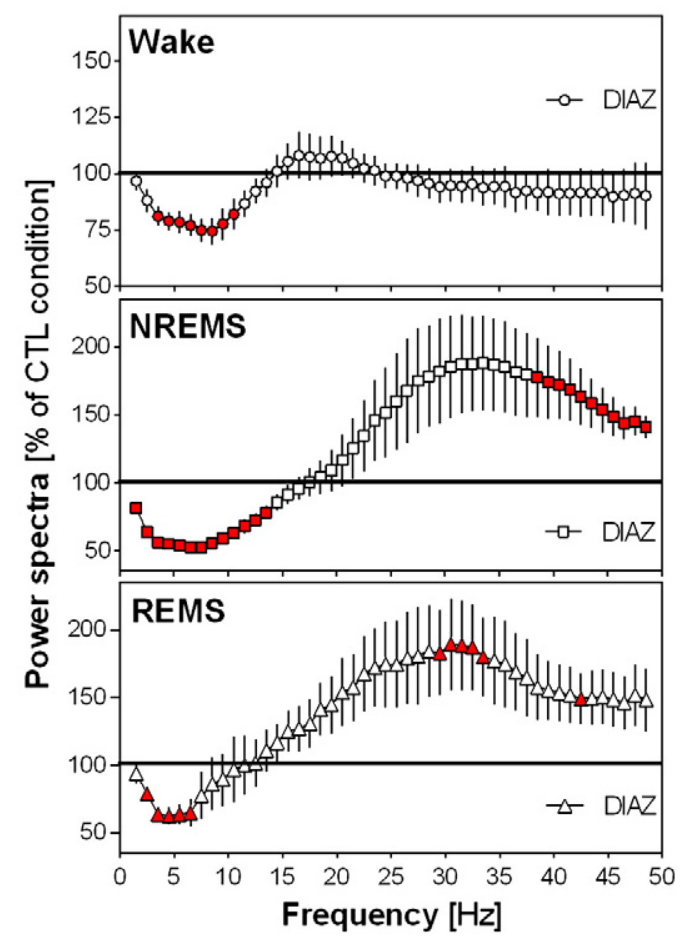

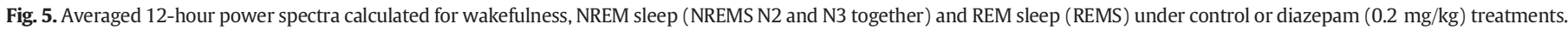

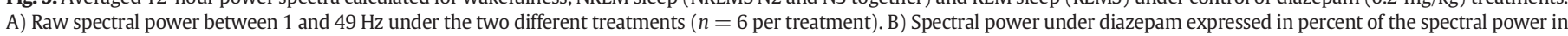
each $\mathrm{Hz}$-bin during the control treatment. Red symbols indicate significant differences compared to the control condition $(t$-test: $p<0.05)$. 
administration as the total duration of each of the sleep stages was comparable to baseline data obtained prior to d-amphetamine.

Spectral analysis revealed that these changes in sleep stage duration were accompanied with very modest changes in EEG activity (Fig. 2). Indeed, d-amphetamine only induced a significant decrease in low EEG frequencies (approximately 4-6 Hz) during wakefulness. In parallel, a slight non-significant decrease in fast EEG activity $(>25 \mathrm{~Hz})$ was observed during stages $\mathrm{N} 2$ and $\mathrm{N} 3$ following d-amphetamine $(1+0.56 \mathrm{mg} / \mathrm{kg}, 9 \mathrm{~h}$ apart $)$. The specificity of these changes for wakefulness might be due to the very low amount of NREM sleep during this part of the recording. Nevertheless, the greatly reduced NREM sleep duration under d-amphetamine was not associated with a compensatory rebound in EEG slow activity during recovery (Fig. 2B).

The administration of lower doses of d-amphetamine (i.e., 0.2 and $0.3 \mathrm{mg} / \mathrm{kg}$ ) revealed a dose-dependent effect on sleep stage duration over the course of a 12 hour (mainly dark) observation period (Fig. 3). In fact, increased time spent awake and decreased N2 and REM sleep were observed only for the $0.3 \mathrm{mg} / \mathrm{kg}$ treatment (Fig. 3A). More specifically, differences were mostly observed during the first half of the dark period and recovered to normal for the second half of the night (Fig. 3B), and thus faster than after the $1 \mathrm{mg} / \mathrm{kg}$ dose (Fig. 1B). Control dosing did not seem to alter duration in any state in comparison to the baseline period with no treatment.

\subsection{Diazepam}

In striking contrast to d-amphetamine, diazepam ( $2 \mathrm{mg} / \mathrm{kg}, \mathrm{PO})$ did not significantly affect sleep stage duration but significantly impacted EEG spectral activity during the different stages over the course of a 12 hour period. Indeed, the slight increase in N2 duration after diazepam administration ( $497 \pm 27$ vs. $463 \pm 32 \mathrm{~min}$ ) and the slight decrease in N3 ( $8 \pm 6$ vs. $35 \pm 20 \mathrm{~min})$ did not reach statistical significance ( $p=0.25$ and $p=0.14$, respectively; Fig. 4). However, spectral analysis revealed a major decrease in the activity in low frequencies (approximately 1-13 Hz) during all vigilance states, including wake, following administration of diazepam (Fig. 5). This effect was strongest regarding NREM sleep for which the frequency range affected was the largest. In addition, diazepam increased EEG activity in high frequencies during sleep. More precisely, spectral power above $37 \mathrm{~Hz}$ was significantly elevated during NREM sleep, while activity around $30 \mathrm{~Hz}$ was significantly elevated during REM sleep.

\subsection{Caffeine}

Caffeine administration produced a dose-dependent effect on both sleep stage duration and EEG activity during vigilance states over the course of the 12 hour observation period. First, 10 and $30 \mathrm{mg} / \mathrm{kg}$ of caffeine significantly increased wakefulness and decreased N2 (Fig. 6A). In addition, the $30 \mathrm{mg} / \mathrm{kg}$ dose significantly reduced REM sleep. For the $10 \mathrm{mg} / \mathrm{kg}$ dose, these effects were observed for the first $4 \mathrm{~h}$ after caffeine administration whereas the effects remained for the full dark period for the $30 \mathrm{mg} / \mathrm{kg}$ dose (Fig. 6B). Administration of $3 \mathrm{mg} / \mathrm{kg}$ of caffeine did not significantly affect the duration of sleep stages.

Caffeine induced changes in EEG activity mainly during wakefulness and NREM sleep (Fig. 7). During wakefulness, higher doses of caffeine (i.e. 10 and $30 \mathrm{mg} / \mathrm{kg}$ ) markedly reduced low frequency EEG activity $(<20 \mathrm{~Hz})$. During NREM sleep, the lowest and the highest doses of caffeine increased high frequency EEG activity $(>20 \mathrm{~Hz}$ ), with the low dose (i.e. $3 \mathrm{mg} / \mathrm{kg}$ ) inducing considerable increases (approximately $150-300 \%$ of spectral power compared to the control treatment). REM sleep spectral activity below $5 \mathrm{~Hz}$ was increased by $3 \mathrm{mg} / \mathrm{kg}$ but decreased by 10 or $30 \mathrm{mg} / \mathrm{kg}$ of caffeine. Overall, these observations indicate a complex pattern of EEG spectral activity in response to different dosage of caffeine, which depends on vigilance state.

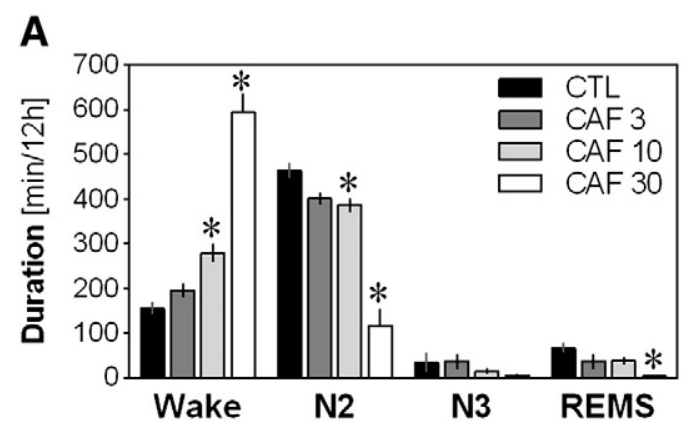

B

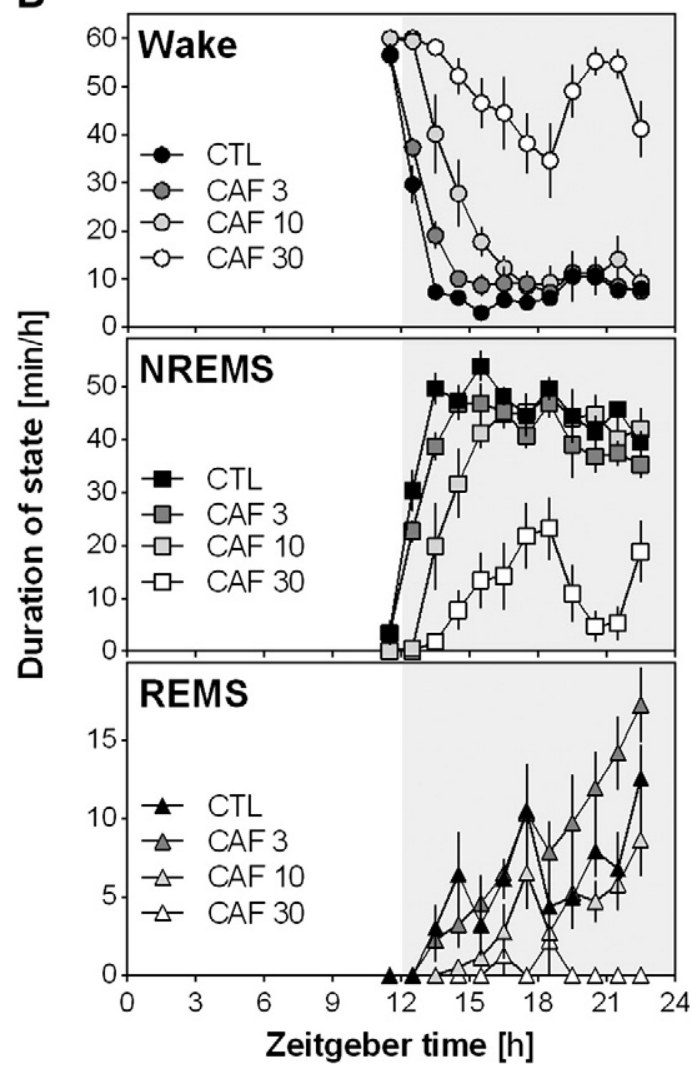

Fig. 6. Duration and distribution of vigilance states in Cynomolgus monkeys under control treatment or three different doses of caffeine $(3,10$ and $30 \mathrm{mg} / \mathrm{kg})$. A) Total duration of wakefulness, N2, N3 and REM sleep (REMS) for a 12-hour period averaged for 5 monkeys. Caffeine significantly changed wakefulness, N2 and REMS $\left(\mathrm{F}_{3,12}>3.9, p<0.05\right)$. Stars indicate Tukey HSD $<0.05$ compared to control. B) Hourly distribution of wakefulness, NREM sleep (NREMS: N1 to N3) and REMS in control condition, and under caffeine (3,10 and $30 \mathrm{mg} / \mathrm{kg}$ ). Zeitgeber time refers to the time relative to the light/dark schedule with 0 corresponding to lights ON and 12 to lights OFF. Grey backgrounds indicate the dark period.

\section{Discussion}

Numerous factors have increased the relevance of sleep assessments in drug development, and one of these is certainly the profound relation between sleep and mental health (Palagini \& Rosenlicht, 2011). In the present study, we showed that the use of telemetry in Cynomolgus monkeys represents a useful and potentially sensitive non-clinical animal model to study the impact of various pharmacological treatments on both sleep architecture and EEG activity. Indeed, we observed using this model that three pharmacological agents having wellrecognized impacts on sleep parameters, d-amphetamine, diazepam and caffeine, show the expected effects on sleep stage duration and EEG power spectra measured during sleep and wakefulness. Since non-human primates are phylogenetically closer to humans and are 
A

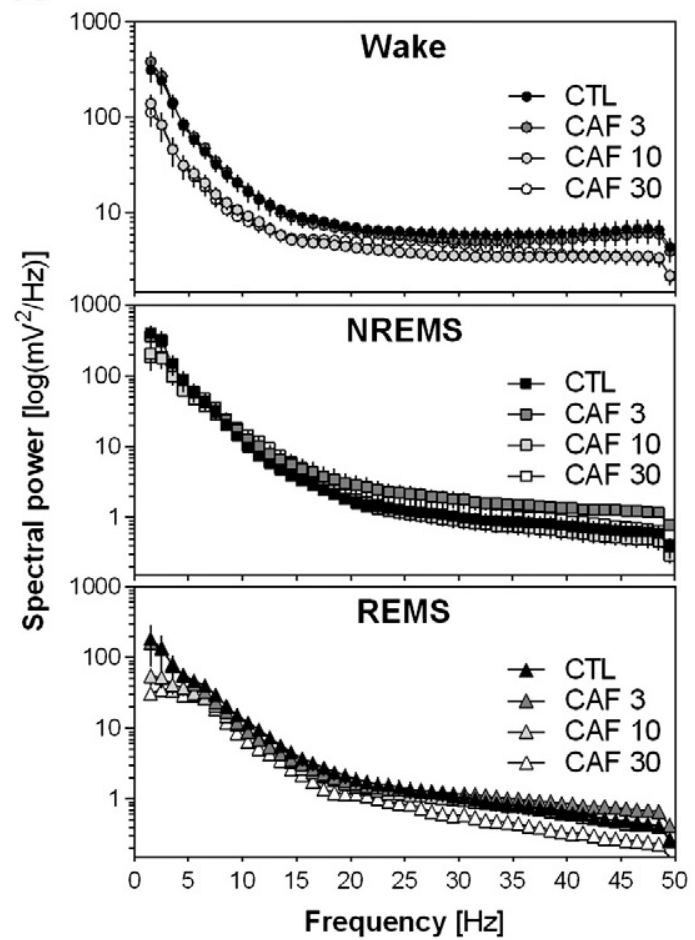

B

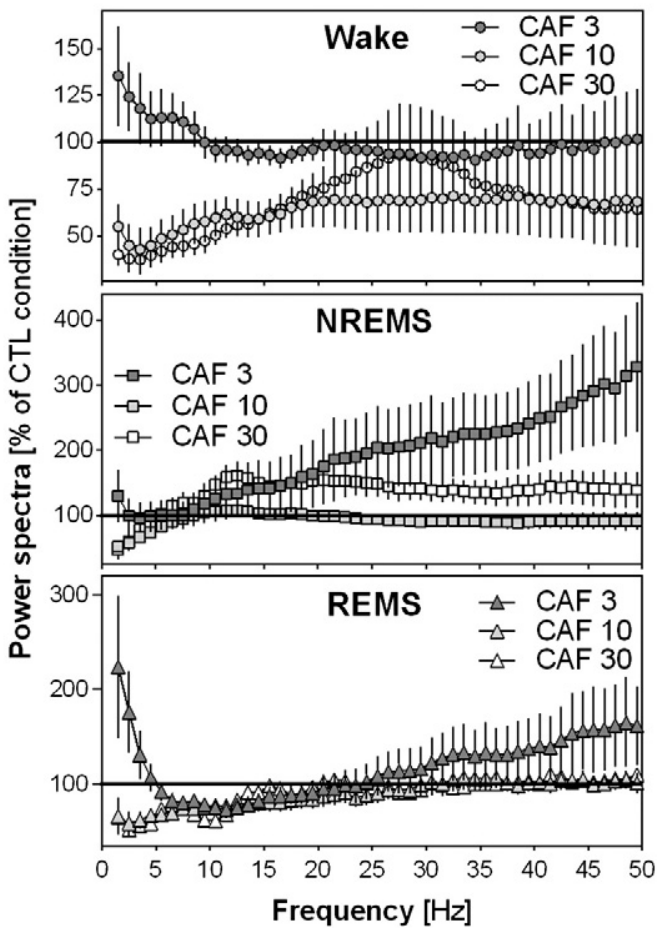

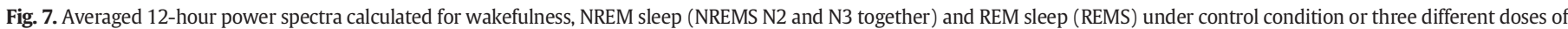

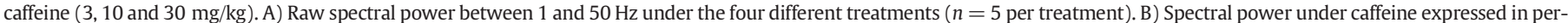
cent of the spectral power in each $\mathrm{Hz}$-bin during the control treatment.

diurnal animals, sleep variables assessed using telemetry in these animals thus present a high translational potential.

Pharmacokinetic parameters in Cynomolgus monkeys are recognized to present a high homology to humans (Ward \& Smith, 2004) increasing the clinical relevance of observations in this species. The characterization of sleep architecture and EEG activity that we recently reported in Cynomolgus monkeys (Rachalski et al., in press) revealed notable similarities with human sleep as described by Carskadon and Dement (2011). For instance, N2 sleep stage was characterized by its distinctive features (i.e., sleep spindles and $\mathrm{K}$ complexes), and REM sleep presented the recognized muscle atonia accompanied with intermittent saccades detected on the EOG. In the present study, baseline and control data, obtained prior to treatment or under control condition, demonstrated that sleep architecture and EEG spectral profile of each sleep stages in Cynomolgus monkeys are similar to those observed in humans. Furthermore, we demonstrate that continuous EEG, EMG and EOG monitoring by telemetry with video in Cynomolgus monkeys was suitable to detect changes following administration of d-amphetamine, diazepam and caffeine, which are reminiscent of changes in sleep duration and spectral activity produced by these substances in humans, as detailed below.

Indeed, administration of d-amphetamine $(1 \mathrm{mg} / \mathrm{kg}, \mathrm{PO})$ increased wakefulness and specifically decreased N2 sleep stage and REM sleep. These effects were dose-dependent as a mid dose $(0.3 \mathrm{mg} / \mathrm{kg})$ significantly increased wakefulness and decreased N2 sleep stage and REM sleep with a lower magnitude than the high dose $(1 \mathrm{mg} / \mathrm{kg})$, whereas the lowest dose $(0.2 \mathrm{mg} / \mathrm{kg})$ did not significantly alter sleep parameters. This supports the use of moderate to low dose when using this agent as a positive control. These effects were similar to those reported in humans (Saletu et al., 1989). Our observation of an absence of delta activity rebound during NREM sleep after d-amphetamine administration is also consistent with previous report in humans (Chapotot, Pigeau, Canini, Bourdon, \& Buguet, 2003). In contrast, administration of diazepam did not significantly alter sleep duration in the healthy animals studied but significantly impacted EEG spectral activity during
Wake, NREMS and REMS. Our observation of a decrease in delta and theta activity $(1-8 \mathrm{~Hz}$ ) and increase in high beta/gamma activity $(>30 \mathrm{~Hz})$ under diazepam is also similar to what has been reported during both wakefulness and sleep in humans (Feng \& Gu, 2005; Hall, Barnes, Furlong, Seri, \& Hillebrand, 2010).

In addition, we observed that caffeine dose-dependently reduced sleep time in Cynomolgus monkeys with a pharmacodynamic profile matching expected pharmacokinetics of this agent (Bonati, Latini, Tognoni, Young, \& Garattini, 1984). More precisely, as expected, caffeine increased time spent awake and decreased N2 sleep and REM sleep during the 12-hour dark period. In addition, caffeine administration changed EEG activity in a state- and dose-dependent manner, with reduced low frequency activity during wakefulness and elevated high frequency during NREM sleep. These changes both in sleep duration and EEG activity following caffeine administration are again consistent with observations regarding the impact of this substance on human sleep and EEG synchrony (Carrier et al., 2007; Drapeau et al., 2006; Landolt et al., 2004).

Several factors can modify the sleep/wake cycle and sleep architecture, such as age, gender, diet, physical activity, concurrent diseases and stress. For instance, time spent in N3 and REM sleep tends to decrease in early adolescence. By late adolescence, N3 sleep may decrease by $40 \%$ and may disappear completely in older individuals (Carskadon \& Dement, 2011). Indeed, total sleep time, sleep efficiency, slow wave sleep and REM sleep decrease with age, while sleep latency, N1 and N2 sleep and wake after sleep onset increase with age (Ohayon, Carskadon, Guilleminault, \& Vitiello, 2004). Accordingly, sleep architecture measurements from the present study obtained from adult Cynomolgus monkeys should mostly be indicative of the typical sleep pattern observed in human adults and may not necessarily apply to pediatric or geriatric populations, whose sleep/wake cycle significantly differs from those of adults.

The increasing knowledge regarding the physiological roles of sleep in brain and organism function increases the relevance of sleep assessments in drug development. Indeed, drug-induced sleep disturbances may have long-term consequences on human health and thus require 
appropriate investigations during development, particularly prior to clinical trials. Moreover, with the aging population, the incidence of sleep apnea has significantly increased over the last two decades (Levy, Tamisier, Minville, Launois, \& Pepin, 2011), in addition to the average $30 \%$ of adults that will report experiencing some symptoms of insomnia over a lifetime (Roth, 2007). In this context, increasing efforts need to be deployed to identify and manage drug-induced sleep disorders throughout the pharmaceutical development cycle to maximally protect populations already at risk of suffering from disrupted sleep. The decision to conduct polysomnography investigations with animal models during drug development is typically based on pharmacological considerations including the drug class, the indication, the pharmacokinetics, prior observations in toxicology or even clinical studies. For indications that require life-long treatment (e.g. neurodegenerative disorders, analgesia, Parkinson's disease), alterations in polysomnography-derived variables may have a major impact on the commercial value of the drug product. In conclusion, our results support the use of continuous EEG, EMG, and EOG monitoring using telemetry in Cynomolgus monkeys as a valuable tool to evaluate drug-induced changes in sleep time and microarchitecture, and also to assess sedative and stimulatory drug effects.

\section{Conflicts of interest}

None of the authors have any conflicts of interest, other than their employment in commercial pharmaceutical companies or contract research organizations (SA, LB, MP, DP).

\section{References}

Authier, S., Paquette, D., Gauvin, D., Sammut, V., Fournier, S., Chaurand, F., et al. (2009). Video-electroencephalography in conscious non human primate using radiotelemetry and computerized analysis: Refinement of a safety pharmacology model. Journal of Pharmacological and Toxicological Methods, 60, 88-93.

Baker, F. C., Mitchell, D., \& Driver, H. S. (2001). Oral contraceptives alter sleep and raise body temperature in young women. Pflügers Archiv, 442, 729-737.

Barraud, Q., Lambrecq, V., Forni, C., McGuire, S., Hill, M., Bioulac, B., et al. (2009). Sleep disorders in Parkinson's disease: The contribution of the MPTP non-human primate model. Experimental Neurology, 219, 574-582.

Benca, R. M., Obermeyer, W. H., Shelton, S. E., Droster, J., \& Kalin, N. H. (2000). Effects of amygdala lesions on sleep in rhesus monkeys. Brain Research, 879, 130-138.

Betts, T. A., \& Alford, C. (1985). Beta-blockers and sleep: A controlled trial. European Journal of Clinical Pharmacology, 28, suppl: 65-68.

Bonati, M., Latini, R., Tognoni, G., Young, J. F., \& Garattini, S. (1984). Interspecies comparison of in vivo caffeine pharmacokinetics in man, monkey, rabbit, rat, and mouse. Drug Metabolism Reviews, 15(7), 1355-1383.

Bonnet, M. H., \& Arand, D. L. (1992). Caffeine use as a model of acute and chronic insomnia. Sleep, 15, 526-536.

Boutrel, B., \& Koob, G. F. (2004). What keeps us awake: The neuropharmacology of stimulants and wakefulness-promoting medications. Sleep, 27, 1181-1194.

Buscemi, N., Vandermeer, B., Friesen, C., Bialy, L., Tubman, M., Ospina, M., et al. (2005). Manifestations and management of chronic insomnia in adults. Evidence report/technology assessment: number 125. Agency for Healthcare Research and Quality.

Campbell, I. G. (2009). EEG recording and analysis for sleep research. Current Protocols in Neuroscience, 49(10.2), 10.2.1-10.2.19.

Carlstedt, R. A. (2009). Handbook of integrative clinical psychology, psychiatry, and behavioral medicine: Perspectives, practices, and research. Springer Publishing Company, 128-130.

Carrier, J., Fernandez-Bolanos, M., Robillard, R., Dumont, M., Paquet, J., Selmaoui, B., et al (2007). Effects of caffeine are more marked on daytime recovery sleep than on nocturnal sleep. Neuropsychopharmacology, 32, 964-972.

Carskadon, M. A., \& Dement, W. C. (2011). Monitoring and staging human sleep. In M. H. Kryger, T. Roth, \& W. C. Dement (Eds.), Principles and practice of sleep medicine (pp. 16-26) (5th ed.). St. Louis: Elsevier Saunders.

Chapotot, F., Pigeau, R., Canini, F., Bourdon, L., \& Buguet, A. (2003). Distinctive effects of modafinil and d-amphetamine on the homeostatic and circadian modulation of the human waking EEG. Psychopharmacology, 166, 127-138.

Chen, W., \& Black, J. (2005). Quantitative analysis of the sleep electroencephalogram. In C. Guilleminault (Ed.), Clinical neurophysiology of sleep disorders (pp. 103-124). Amsterdam, The Netherlands: Elsevier Health Sciences.

Drapeau, C., Hamel-Hébert, I., Robillard, R., Selmaoui, B., Filipini, D., \& Carrier, J. (2006). Challenging sleep in aging: the effects of $200 \mathrm{mg}$ of caffeine during the evening in young and middle-aged moderate caffeine consumers. Journal of Sleep Research, 15, 133-141.
European Medicines Agency (2011). Guideline on medicinal products for the treatment of insomnia. http://www.ema.europa.eu/docs/en_GB/document_library/Scientific guideline/2011/02/WC500102351.pdf

Feng, Z., \& Gu, F. (2005). Power spectral analysis of recovery sleep of sleep deprivation and hypnotic drug induced sleep. Conference Proceedings-IEEE Engineering in Medicine and Biology Society, 4, 3593-3596.

Franken, P., Malafosse, A., \& Tafti, M. (1998). Genetic variation in EEG activity during sleep in inbred mice. American Journal of Physiology. Regulatory, Integrative and Comparative Physiology, 275, R1127-R1137.

Gottlieb, D. J., Punjani, N. M., Newman, A. B., Resnick, H. E., Redline, S., Baldwin, C. M., et al. (2005). Association of sleep time with diabetes mellitus and impaired glucose tolerance. Archives of Internal Medecine, 165, 863-867.

Hall, S. D., Barnes, G. R., Furlong, P. L., Seri, S., \& Hillebrand, A. (2010). Neuronal network pharmacodynamics of GABAergic modulation in the human cortex determined using pharmaco-magnetoencephalography. Human Brain Mapping, 31, 581-594.

Hasan, S., Pradervand, S., Ahnaou, A., Drinkenburg, W., Tafti, M., \& Franken, P. (2009). How to keep the brain awake? The complex molecular pharmacogenetics of wake promotion. Neuropsychopharmacology, 34, 1625-1640.

Hsieh, K. C., Robinson, E. L., \& Fuller, C. A. (2008). Sleep architecture in unrestrained rhesus monkeys (Macaca mulatta) synchronized to 24-hour light-dark cycles. Sleep, $31,1239-1250$.

Kriegsfeld, L. J., LeSauter, J. L., Hamada, T., Pitts, S. M., \& Silver, R. (2002). Circadian rhythms in the endocrine system. In D. Pfaff, \& A. Etgen (Eds.), Hormones, brain and behavior (pp. 33-91). New York: Academic Press.

Kriegsfled, L. J., \& Silver, R. (2006). The regulation of neuroendocrine function: Timing is everything. Hormones and Behavior, 49, 557-574.

Landolt, H. P., Retey, J. V., Tonz, K., Gottselig, J. M., Khatami, R., Buckelmuller, I., et al. (2004). Caffeine attenuates waking and sleep electroencephalographic markers of sleep homeostasis in humans. Neuropsychopharmacology, 29, 1933-1939.

Levy, P., Tamisier, R., Minville, C., Launois, S., \& Pepin, J. -L. (2011). Sleep apnoea syndrome in 2011: Current concepts and future directions. European Respiratory Review, 20, 134-146.

Mills, E. J., Wu, P., Lockhart, I., Wilson, K., \& Ebbert, J. O. (2010). Adverse events associated with nicotine replacement therapy (NRT) for smoking cessation. A systematic review and meta-analysis of one hundred and twenty studies involving 177,390 individuals. Tobacco Induced Diseases, 8,8 .

Murphy, P. J., Badia, P., Myers, B. L., Boecker, M. R., \& Wright, K. P., Jr. (1994). Nonsteroidal anti-inflammatory drugs affect normal sleep patterns in humans. Physiology $\mathcal{E}^{\circ}$ Behavior, 55, 1063-1066.

Ohayon, M. M., Carskadon, M. A., Guilleminault, C., \& Vitiello, M. V. (2004). Meta-analysis of quantitative sleep parameters from childhood to old age in healthy individuals: Developing normative sleep values across the human lifespan. Sleep, 27, 1255-1273.

Palagini, L., \& Rosenlicht, N. (2011). Sleep, dreaming, and mental health: A review of historical and neurobiological perspectives. Sleep Medicine Reviews, 15, 179-186.

Palma, J. A., Urrestarazu, E., \& Iriarte, J. (2013). Sleep loss as risk factor for neurological disorders: A review. Sleep Medicine, 14, 229-236.

Paterson, L. M., Wilson, S. J., Nutt, D. J., Hutson, P. H., \& Ivarsson, M. (2009). Characterisation of the effects of caffeine on sleep in the rat: a potential model of sleep disruption. Journal of Psychopharmacology, 23, 475-486.

Pigarev, I. N., Almirall, H., \& Pigareva, M. L. (2008). Cortical evoked responses to magnetic stimulation of macaque's abdominal wall in sleep-wake cycle. Acta Neurobiologiae Experimentalis, 68, 91-96.

Porsolt, R. D. (2013). The usefulness of non-human primates in central nervous system safety pharmacology. Journal of Pharmacological and Toxicological Methods, 68, 23-29.

Rachalski, A., Authier, S., Bassett, L., Pouliot, M., Tremblay, G., \& Mongrain, V. (2014). Sleep electroencephalographic characteristics of the Cynomolgus monkey measured by telemetry. Journal of Sleep Research (in press).

Ramakrishnan, K., \& Scheid, D. C. (2007). Treatment options for insomnia. American Family Physician, 76, 517-526.

Roth, T. (2007). Insomnia: Definition, prevalence, etiology, and consequences. Journal of Clinical Sleep Medicine, 3, S7-S10.

Saletu, B., Frey, R., Krupka, M., Anderer, P., Grünberger, J., \& Barbanoj, M. J. (1989). Differential effects of a new central adrenergic agonist-modafinil-and D-amphetamine on sleep and early morning behaviour in young healthy volunteers. International Journal of Clinical Pharmacology Research, 9, 183-195.

Schmid, D. A., Wichniak, A., Uhr, M., Ising, M., Brunner, H., Held, K., et al. (2006). Changes of sleep architecture, spectral composition of sleep EEG, the nocturnal sectretion of cortisol, ACTH, GH, prolactin, melatonin, ghrelin, and leptin, and the DEX-CRH test in depressed patients during treatment with mirtazapine. Neuropsychopharmacology, 31, 832-844.

Tononi, G., \& Cirelli, C. (2006). Sleep function and synaptic homeostasis. Sleep Medicine Reviews, 10, 49-62.

Van Twyver, H. (1969). Sleep patterns of five rodent species. Physiology \& Behavior, 4, 901-905.

Vgontzas, A. N., Mastorakos, G., Bixler, E. O., Kales, A., Gold, P. W., \& Chrousos, G. P. (1999). Sleep deprivation effects on the activity of the hypothalamic-pituitary-adrenal and growth axes: Potential clinical implications. Clinical Endocrinology (Oxford), 51, 205-215.

Ward, K. W., \& Smith, B. R. (2004). A comprehensive quantitative and qualitative evaluation of extrapolation of intravenous pharmacokinetic parameters from rat, dog, and monkey to humans. II. Volume of distribution and mean residence time. Drug Metabolism and Disposition, 32, 612-619.

Zhdanova, I. V., Geiger, D. A., Schwagerl, A. L., Leclair, O. U., Killiany, R., Taylor, J. A., et al. (2002). Melatonin promotes sleep in three species of diurnal nonhuman primates. Physiology \& Behavior, 75, 523-529. 